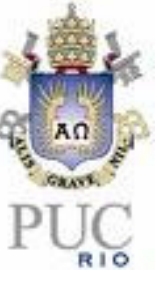

Fabio Veloso Vicente da Silva

\title{
Análise das Estratégias Competitivas na Indústria Automobilística
}

Dissertação de Mestrado

Dissertação apresentada como requisito parcial para obtenção do título de Mestre pelo Programa de Pós-Graduação no Departamento de Administração da PUC-Rio.

Orientador: Prof: Jorge Ferreira da Silva

Rio de Janeiro Maio de 2007 


\section{Fabio Veloso Vicente da Silva}

\section{Análise das Estratégias Competitivas na Indústria Automobilística}

Dissertação apresentada como requisito parcial para obtenção do grau de Mestre pelo Programa de Pósgraduação em Administração de Empresas da PUC-Rio.

Aprovada pela Comissão Examinadora abaixo assinada.

Prof. Jorge Ferreira da Silva

Orientador

Departamento de Administração - PUC-Rio

Prof. Hélène Bertrand

Departamento de Administração - PUC-Rio

Prof. â Ângela Maria Cavalcanti da Rocha

UFRJ

Prof. João Pontes Nogueira

Vice-Decano de pós-graduação do CCS

Rio de Janeiro, 22 de maio de 2007. 
Todos os direitos reservados. É proibida a reprodução total ou parcial do trabalho sem a autorização da universidade, do autor e do orientador.

Fabio Veloso Vicente da Silva

Graduou-se em Engenharia de Produção pela Universidade Federal Fluminense (UFF) em 2003.

Ficha Catalográfica

Silva, Fabio Veloso Vicente da

Análise das estratégias competitivas dentro da indústria automobilística / Fabio Veloso Vicente da Silva; orientador: Jorge Ferreira da Silva. - 2007.

124 f. il. ; $30 \mathrm{~cm}$

Dissertação (Mestrado em Administração)-Pontifícia Universidade Católica do Rio de Janeiro, Rio de Janeiro, 2008.

Inclui bibliografia

1. Administração - Teses. 2. Estratégia. 3. Grupos estratégicos. 4. Tipologia de Porter. 5. Análise de desempenho. 6. Indústria automobilística. I. Silva, Jorge Ferreira da. II. Pontifícia Universidade Católica do Rio de Janeiro. Departamento de Administração. III. Título.

CDD: 658 
Para meus pais, Selma e Mauro, pelo apoio e confiança, e também a toda minha família. 


\section{Agradecimentos}

Ao Professor Jorge Ferreira da Silva, por sua orientação e contribuição para o aperfeiçoamento da minha formação acadêmica ao longo do curso e especialmente na realização deste trabalho.

Aos meus pais Mauro Sebastião e Selma, e aos meus avós Sebastião e Maria Thereza, pelo amor, carinho, apoio e dedicação de forma incondicional, além da transmissão de valores que foram e estão sendo fundamentais ao longo de minha vida.

A Maíra Fortes de Lima e meus amigos pela compreensão da minha ausência em muitos momentos devido a compromissos exigidos pelo curso. 


\section{Resumo}

Silva, Fabio Veloso Vicente da; Silva, Jorge Ferreira, Análise das Estratégias Competitivas na Indústria Automobilística. Rio de Janeiro, 2007. 124 p. Dissertação de Mestrado - Departamento de Administração, Pontifícia Universidade Católica do Rio de Janeiro.

Este trabalho teve como principal objetivo o estudo da indústria automobilística brasileira, relacionando o posicionamento estratégico competitivo das empresas com a formação de grupos estratégicos e o desempenho dos mesmos. O período estudado compreende os anos de 2001 a 2006, a amostra foi formada por 20 empresas ligadas à Abeiva - Associação Brasileira de Importadores Veículos Automotores - e à Anfavea Associação Nacional dos Fabricantes de Veículos Automotores, e a análise foi baseada na tipologia de Porter. Os resultados demonstraram que empresas que apresentam melhores desempenhos são as que optaram por uma das estratégias genéricas propostas por Porter, no caso a de Diferenciação; as empresas que obtiveram os piores resultados foram as que não apresentaram estratégia definida (stuck-in-the-middle).

\section{Palavras-chave}

Estratégia; Grupos Estratégicos, Tipologia de Porter, Análise de Desempenho, Indústria Automobilística. 


\section{Abstract}

Silva, Fabio Veloso Vicente da; Silva, Jorge Ferreira, Strategy analysis of the automobile industry. Rio de Janeiro, 2007. 124 p. Master dissertation Administration Department, Pontifícia Universidade Católica do Rio de Janeiro.

This research aims to study the Brazilian automobile industry, relating the strategic positioning of the companies with the formation of strategic groups and their performances. The studied period started in 2001 and ended in 2006 and the sample was formed by 20 associated companies from Abeiva - the Brazilian Importers Association of Automotive Vehicles and the Anfavea - the National Association of Automotive Vehicles. The study was based on the Porter's typology and the findings showed that the best performances companies are those that applied the generic strategy of Differentiation, moreover, the companies who had gotten the worse result had been the ones who had not presented a well-defined strategy (stuck-in-the-middle).

Keywords:

Strategy; Strategic Groups, Porter's Typology, Performance Analysis, Automobile Industry. 


\section{Sumário}

1. O problema 15

1.1. Introdução 15

1.2. Objetivos da pesquisa 17

$\begin{array}{ll}\text { 1.2.1. Objetivos intermediários } & 17\end{array}$

1.3. Delimitação do estudo 18

1.4. Relevância do estudo 18

1.5. Estrutura do trabalho 19

2. Referencial teórico 20

2.1. Introdução 20

2.2. Definições centrais 22

2.2.1. Estratégia $\quad 22$

2.2.2. Vantagem competitiva 24

2.2.3. Grupos estratégicos 25

2.3. A estratégia e o ambiente 26

2.4. Desempenho 28

2.4.1. Avaliação do desempenho 30

2.4.2. Desempenho entre grupos estratégicos 30

2.4.3. Desempenho dentro de grupos estratégicos 31

2.4.4. Critérios competitivos 33

2.5. Modelo das cinco forças 33

2.5.1. Ameaça de novos entrantes 35

2.5.2. Poder de negociação dos fornecedores 36

2.5.3. Poder de negociação dos compradores 37

2.5.4. Ameaça de produtos substitutos 37

2.5.5. Rivalidade entre concorrentes 38 
2.6. Tipologias estratégicas $\quad 40$

2.6.1. Tipologia de Porter $\quad 40$

2.6.1.1. Estratégia de liderança através do custo total 41

2.6.1.2. Estratégia de diferenciação $\quad 42$

2.6.1.3. Estratégia de enfoque 42

2.6.1.4. Stuck-in-the-middle 43

2.6.2. Críticas ao modelo de Porter e novas tipologias 44

2.6.2.1. Críticas à liderança através do custo total 44

2.6.2.2. Crítica à diferenciação 44

2.6.3. Tipologia de Mintzberg 45

2.6.4. Resource-Based View 48

3. Histórico do mercado automobilístico 53

3.1. Histórico brasileiro $\quad 53$

3.1.2. A década de ouro $\quad 54$

3.2. Panorama do mercado nacional - abertura do mercado 56

3.3. Competitividade da indústria automobilística 57

3.4. Posicionamento da indústria automobilística 59

3.5. Tecnologia e futuro $\quad 60$

3.6. Geração de empregos $\quad 61$

3.7. Indústria automobilística mundial 62

3.8. Design $\quad 63$

4. Metodologia 66

4.1. Tipo de pesquisa $\quad 66$

4.2. Universo e amostra 66

$\begin{array}{ll}\text { 4.3. Coleta de dados } & 67\end{array}$

4.3.1. Seleção das variáveis estratégicas 68

4.3.1.1. Política de preço $\quad 68$

$\begin{array}{ll}\text { 4.3.1.2. Foco } & 68\end{array}$

4.3.1.3. Diversificação da linha de produtos 68 
4.3.1.4. Assistência técnica 69

4.3.1.5. Credibilidade da marca 69

$\begin{array}{ll}\text { 4.3.1.6. Propaganda } & 69\end{array}$

4.3.1.7. Melhor compra $\quad 69$

4.3.1.8. Canais de distribuição $\quad 70$

4.3.1.9. Produtos em segmentos high price 70

4.3.1.10. Produtos em segmentos de entrada 70

4.3.2. Seleção das variáveis de desempenho 70

$\begin{array}{ll}\text { 4.3.2.1. Market Share } & 70\end{array}$

4.3.2.2. Market Share ponderado por segmento 71

4.3.2.3. Crescimento do Market Share 71

4.3.2.4. Receita bruta $(\mathrm{R} \$) \quad 71$

4.3.2.5. Preço médio $(\mathrm{R} \$) \quad 71$

4.3.2.6. Satisfação dos consumidores 72

4.4. Tratamento de dados $\quad 72$

4.5. Limitações do método $\quad 73$

$\begin{array}{ll}\text { 5. Análise dos resultados } & 75\end{array}$

5.1. Análise da normalidade das variáveis 75

5.2. Análise de correlações 76

5.3. Redução do espaço estratégico

5.4. Formação dos grupos estratégicos $\quad 84$

5.5.Apresentação dos grupos estratégicos $\quad 88$

5.5.1. Análise dos grupos estratégicos 89

5.5.1.1. Grupo 1: Liderança através do custo total 90

5.5.1.2. Grupo 2: Diferenciação 91

5.5.1.3. Grupo 3: Enfoque em custo 93

5.5.1.4. Grupo 4: Enfoque em diferenciação 93

5.5.1.5. Grupo 5: Stuck-in-the-Middle 94

5.6. Comparação de desempenho entre grupos estratégicos 95 
$\begin{array}{ll}\text { 6. Conclusão } & 101\end{array}$

6.1. Conclusões sobre os resultados da pesquisa 101

6.2. Sugestões para futura pesquisa 117

$\begin{array}{ll}\text { Referência Bibliográfica } & 118\end{array}$

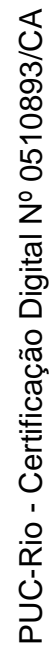




\section{Lista de Tabelas}

Tabela 1 - Alternativas de medição de desempenho. 32

Tabela 2 - Principais barreiras de entradas 39

$\begin{array}{ll}\text { Tabela } 3 \text { - Empresas participantes do estudo } & 67\end{array}$

Tabela 4 - Teste de normalidade das variáveis de desempenho 75

Tabela 5 - Teste de Kolmogorov - Smirnov das variáveis de desempenho 75

Tabela 6 - Teste de normalidade das variáveis estratégicas 76

Tabela 7 - Teste de Kolmogorov - Smirnov das variáveis estratégicas 76

Tabela 8 - Matriz de correlação de Pearson das variáveis estratégicas 77

Tabela 9 - Matriz de correlação de Pearson das variáveis de desempenho 80

Tabela 10 - Resultado da análise de fatores $\quad 81$

Tabela 11 - Carga final dos fatores após rotação nos eixos 82

Tabela 12 - Fatores resultantes da análise das variáveis estratégicas 84

Tabela 13 - Percentis utilizados para a montagem da matriz inicial dos 85

centróides

Tabela 14 - Matriz de centróides iniciais - distribuição teórica 86

Tabela 15 - Matriz de centróides iniciais - distribuição dos percentis 86

aplicados

Tabela 16 - Matriz final de centróides $\quad 87$

Tabela 17 - Teste de Wilcoxon $\quad 87$

Tabela 18 - Matriz divisão por grupo estratégico 88

Tabela 19 - Grupos estratégicos $\quad 89$

Tabela 20 - Matriz divisão por grupo estratégico 90

Tabela 21 - Teste de igualdade de desempenho dos grupos estratégicos 95

Tabela 22 - Comparação de desempenho entre grupos estratégicos - teste de 96

Bonferroni

Tabela 22b - Continuação da tabela 22 
Tabela 23 - Classificação geral das variáveis de desempenho 


\section{Lista de Figuras}

Figura 1 - Organização e ambiente 26

Figura 2 - Modelo de Andrews e Christensen 27

Figura 3 - Perspectiva de Porter 29

Figura 4 - Perspectiva neo-austríaca $\quad 29$

Figura 5 - As cinco forças de Porter 34

Figura 6 - As estratégias genéricas de Porter (1980) 40 